\title{
Monitoring of Conductivity Changes in Passive Layers by Scanning Electrochemical Microscopy in Feedback Mode: Localization of Pitting Precursor Sites on Surfaces of Multi-Metallic Phase Materials
}

\author{
Marcin A. Malik ${ }^{1, *}$ and Pawel J. Kulesza ${ }^{2}$
}

\begin{abstract}
${ }^{1}$ Division of Chemistry, Department of Materials Processing Engineering and Applied Physics, Częstochowa University of Technology, Al. Armii Krajowej 19, 42-201 Częstochowa, Poland

${ }^{2}$ Department of Chemistry, University of Warsaw, Pasteura 1, 02-093 Warsaw, Poland
\end{abstract}

\section{SUPPORTING INFORMATION}

\section{CONTENT}

Detailed description of experimental setup used for SECM imaging. Characteristics of Fe-inclusions in the $\mathrm{Nd}_{13.5} \mathrm{Fe}_{79.5} \mathrm{Si}_{1} \mathrm{~B}_{6}$ magnet: SEM images, EDX maps of surface concentration of elements (Fe, $\mathrm{Nd}$, O) and EDX spectra acquired from the bulk material as well as from a Fe-inclusion. Results of model experiments concerning resolution and contrast in SECM: images of $25 \mu \mathrm{m}$ Pt wire embedded in glass insulating sheath obtained at different tip-substrate distances; and comparison of approach curves recorded over an insulating glass sheath and over Pt micro-wire. Optical micrographs of a pit developed on the surface of $\mathrm{Nd}_{13.5} \mathrm{Fe}_{79.5} \mathrm{Si}_{1} \mathrm{~B}_{6}$ after passive layer breakdown. Cyclic voltammograms for iron electrode in phosphate buffer solutions. Admittance vs potential plot for iron electrode in phosphate buffer. Electrochemical impedance spectra recorded during prolonged exposure of iron electrode to 0.1 mol dm $\mathrm{dm}^{-3}$ phosphate buffer at $\mathrm{pH}=7$.

To whom correspondence should be addressed. Phone/Fax: +48 343250705 . E-mail: malik@mim.pcz.czest.pl. 


\section{Experimental setup used for SECM experiments}
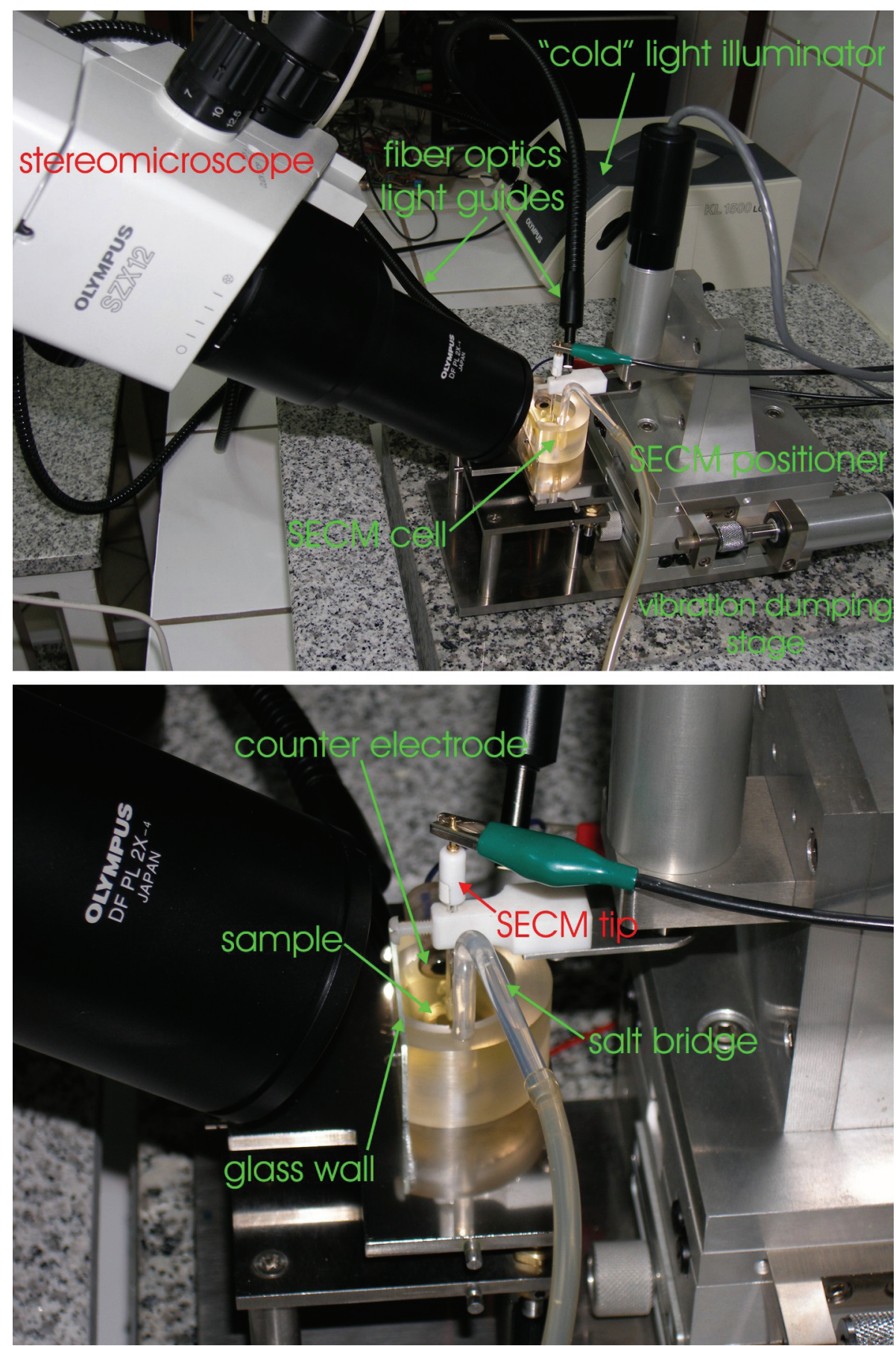

Fig. S-1. The configuration of instruments used during SECM measurements (the top image) and an enlarged view of SECM cell (the bottom image).

Parallel to SECM measurements, the investigated surface of the $\mathrm{Nd}_{13.5} \mathrm{Fe}_{79.5} \mathrm{Si}_{1} \mathrm{~B}_{6}$ magnet (immersed in a phosphate buffer solution) was inspected using a stereomicroscope (Model SZX12, Olympus, Japan) equipped with a planachromatic objective DF PL 2X (numerical aperture, N.A.=0.2) and a digital camera CAMEDIA C5060-WZ. A cold light source (Olympus, KL 1500 LCD) with light guides was used to perform oblique observations of the investigated surface using stereomicroscope. The 
SECM cell housing (made of Plexiglas) was equipped with a glass wall in a form of a standard microscope slide (stuck to the housing by silicone glue). The theoretical resolving power of a light microscope with an objective having N.A. $=0.2$ (used in our setup) is equal to ca. $1.5 \mu \mathrm{m}$ [S1a]. Even if, in practice, a resolution of our microscope was somewhat lower than the theoretical one, we were able to observe clearly objects having dimensions of tens $\mu \mathrm{m}$. An example is given in Fig. S-5D-E, where the $25-\mu \mathrm{m}$ Pt-wire (embedded in a glass sheath) was clearly visible. Thus it should be emphasized, that using our experimental setup we were able to trace a formation of stable pits (with dimensions of tens $\mu \mathrm{m}$ ) developed on investigated metallic surfaces.

\section{Characteristics of iron inclusions in the $\mathrm{Nd}_{13.5} \mathrm{Fe}_{79.5} \mathrm{Si}_{1} \mathrm{~B}_{6}$ magnet}

The distribution of iron inclusions on the surface of the $\mathrm{Nd}_{13.5} \mathrm{Fe}_{79.5} \mathrm{Si}_{1} \mathrm{~B}_{6}$ magnet is not uniform. Fig. S-2E shows a few Fe inclusions existing close to each other. Single Fe-inclusions also exist in the $\mathrm{Nd}_{13.5} \mathrm{Fe}_{79.5} \mathrm{Si}_{1} \mathrm{~B}_{6}$ magnet. An example of such inclusion is presented in Fig. 1 (the main text). All Feinclusions are irregular in shape. EDX microanalysis of Fe-inclusions reveals that they contain iron at the level of 99.5 at. \%. Examples of EXD spectra characteristic of a Fe-inclusion and the bulk material are presented in Fig. S-2 $(1,2)$. 

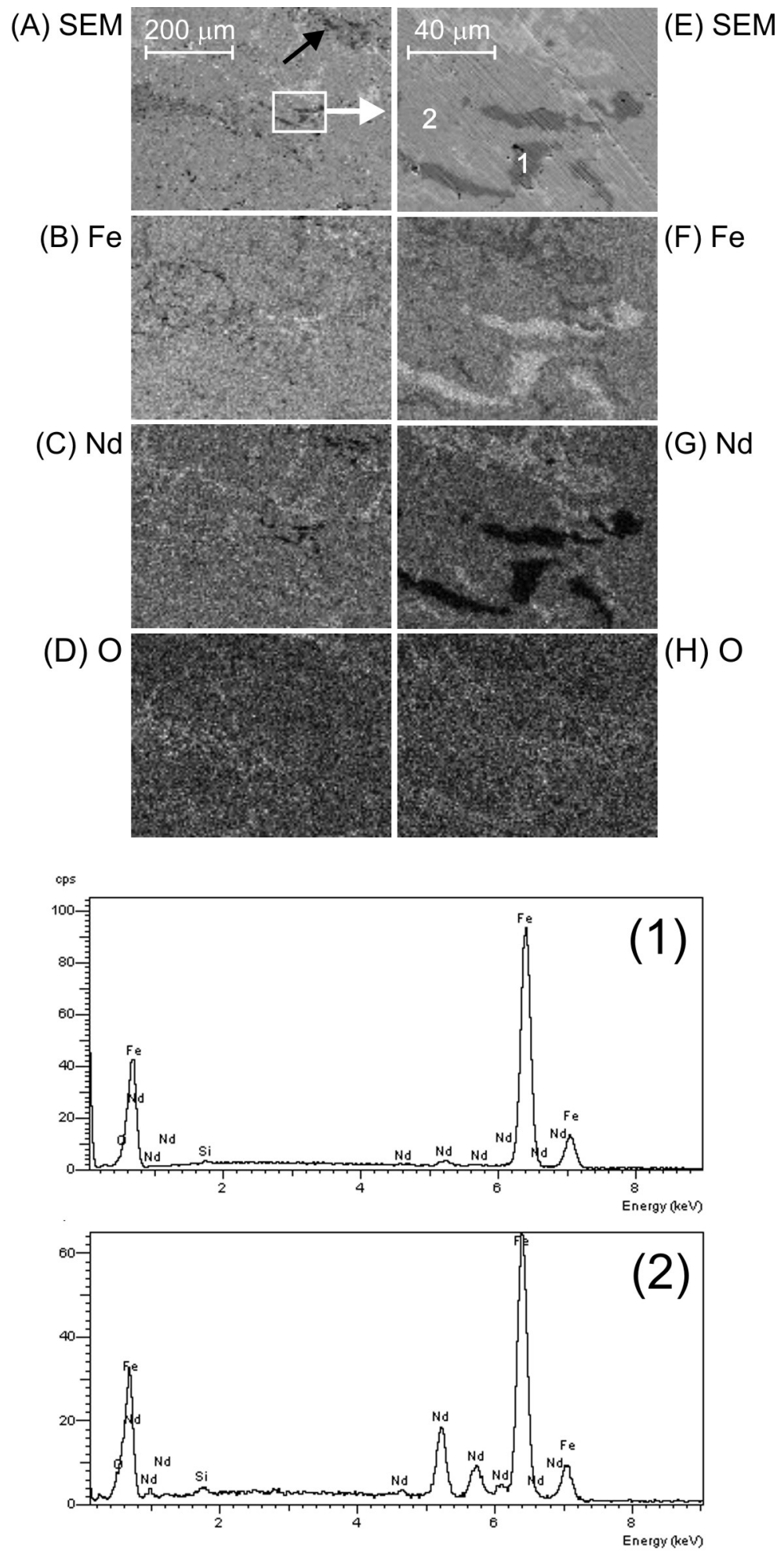

Fig. S-2. SEM micrographs of the surface of $\mathrm{Nd}_{13.5} \mathrm{Fe}_{79.5} \mathrm{Si}_{1} \mathrm{~B}_{6}$ magnet taken at magnifications $200 \mathrm{x}$ (A) and 1000x (E). SEM images and respective surface concentration maps (obtained by EDX) are shown below for $\mathrm{Fe}(\mathrm{B}, \mathrm{F}), \mathrm{Nd}(\mathrm{C}, \mathrm{G})$ and $\mathrm{O}(\mathrm{D}, \mathrm{H})$. Darker areas on images $(\mathrm{B}-\mathrm{H})$ reflect lower concentrations of elements. EDX spectra, which were recorded at spots indicated by numbers $(1,2)$ on the image $(E)$, are presented at the bottom. The area in white rectangle on the image (A) is illustrated at higher magnification in comparison to the image $(\mathrm{E})$. The Fe-inclusion indicated by a black arrow on the image (A) is shown as Fig. 1 in the main text of our paper. 
SECM model experiments - imaging of Pt-wire of $25-\mu \mathrm{m}$ diameter embedded in glass at different tip-substrate distances
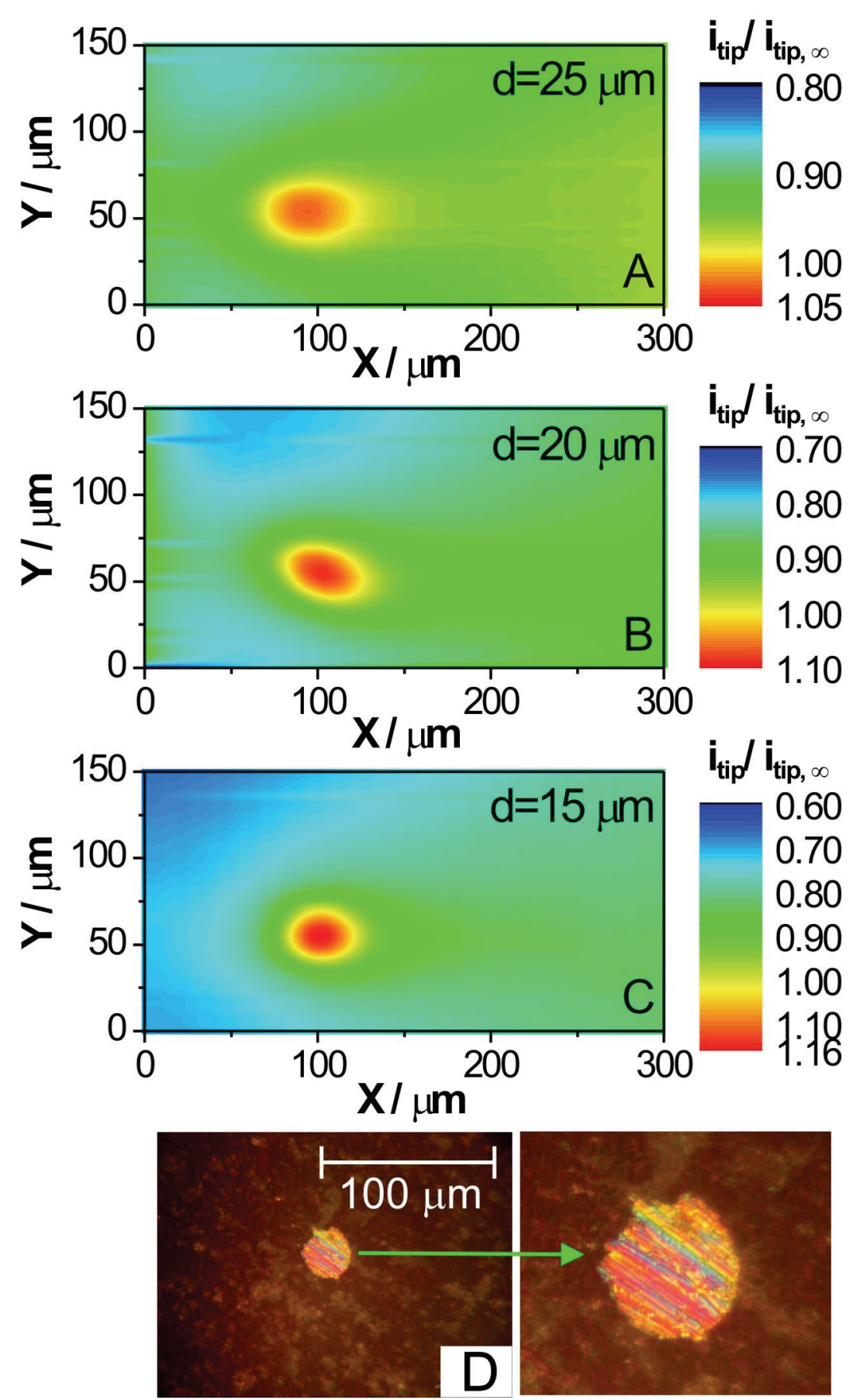

Fig. S-3. SECM images (A-C) acquired for the model substrate - the $25 \mu \mathrm{m}$ (diameter) Pt wire embedded in glass. Electrolyte: $2 \mathrm{mmol} \mathrm{dm}^{-3} \mathrm{FcCOOH}$ in $0.1 \mathrm{~mol} \mathrm{dm}^{-3}$ phosphate buffer at $\mathrm{pH}=7$. $E_{t i p}=0.5 \mathrm{~V}$ vs SCE. $E_{s u b}=-0.3 \mathrm{~V}$ vs SCE. Tip-substrate distances $(d)$ are labeled on respective images. Pt tip microelectrode diameter: $25 \mu \mathrm{m}(\mathrm{RG}=8)$. Tip movement rate: $50 \mu \mathrm{m} \mathrm{s}^{-1}$. Differential interference contrast (DIC) optical micrograph (D) of the substrate used for SECM imaging. The area with Pt microwire is enlarged at the right sight of the micrograph (D). Surface imperfections (ca. $1 \mu \mathrm{m}$ in height) revealed by DIC optical microscopy (scratches after substrate polishing on emery paper grid no. 2500) were not detected by SECM with $25 \mu \mathrm{m}$ tip. 
In general, SECM is capable to distinguish conducting objects of the sizes comparable to the microelectrode tip radius from the insulating matrix. We show in Fig. S-3 results of the model SECM imaging experiments performed on the $25-\mu \mathrm{m}$ Pt-wire embedded in glass sheath as a model substrate. It is noteworthy that the $25-\mu \mathrm{m}$ Pt-microelectrode tip (with $\mathrm{RG}=8$ ) was used in these experiments. SECM images were acquired at three selected tip-substrate distances, namely $d=25,20$ and $15 \mu \mathrm{m}$. The tipsubstrate distances were assigned from the approach curves recorded over an insulating part of the substrate. In practice, the tip was positioned at least $200 \mu \mathrm{m}$ far from the conducting spot (here $\mathrm{Pt}$ microwire). An example of an approach curve recorded over insulating glass sheath is presented in Fig. S-4A. It should be stressed that the experimental curve perfectly overlaps the theoretical one. Thus precise assignment of the tip-substrate distance can be achieved by monitoring current of the tip during its moving to the glass surface (and halting it when the tip current reaches a certain value predicted by the theory for the desired value of $d$ ).

It comes from the data of Fig. S-3 that a conducting circular object (having diameter of $25 \mu \mathrm{m}$ and placed within an insulating matrix) can be easily monitored and observed at SECM images recorded with use of the $25-\mu \mathrm{m}$ microelectrode-tip. Obviously, the observation is influenced by certain experimental factors. First, the smaller tip-substrate distance the sharper image can be obtained, and the observed dimensions of an investigated object are closer to the real one. Visible dimensions (measured in horizontal direction) of the object are 52,41 and $36 \mu \mathrm{m}$ for the tip-substrate distances equal to 25,20 and $15 \mu \mathrm{m}$, respectively (Fig. S-3, A-C). Second, the circular object appears as an ellipse, and this deviation is due to the horizontal tip movement during scanning. Consequences of hydrodynamics (generated by the motion of a scanning tip) have recently been discussed in Ref. [S2].

It would be interesting to approach to the problem of contrast in SECM in a manner analogous to that done in optical microscopy $(\mathrm{OM})$. In OM, the image contrast, $C_{O M}$ (considering gray scale images) is defined as following [S1b]:

$$
C_{O M}=\frac{I_{o b}-I_{b k}}{I_{b k}}
$$


where $I_{b k}$ stands for the background light intensity, and $I_{o b}$ represents light intensity of the object. By analogy, the contrast in SECM, $C_{S E C M}$, can be defined using the following equation:

$$
C_{S E C M}=\frac{i_{o b}-i_{b k}}{i_{b k}}
$$

where $i_{b k}$ is the normalized tip current recorded for the background (here the insulating glass surface), and $i_{o b}$ represents the normalized tip current characteristic of an object (Pt microwire in our model experiments). For $d=25 ; 20$ and $15 \mu \mathrm{m}$, we have calculated the following contrast values $C_{S E C M}=0.29$; 0.53 and 0.84 , respectively. These results show the influence of the tip-substrate distance on the contrast of SECM images. It should be noted that the minimum value of $C_{O M}$ required to distinguish an object from a background is equal to 0.04 in $\mathrm{OM}[\mathrm{S} 1 \mathrm{~b}]$. To better understand the influence of the tip-substrate distance on the contrast in SECM imaging, SECM images (recorded for three different values of $d$ ) are presented in a gray scale (Fig. S-5).

Another factor that influences measurement of the contrast in SECM is conductivity of the investigated object placed in an insulating matrix. A decrease in the object conductivity results effectively in lowering of rate of the mediator's redox reaction (that occurs at the object surface); consequently, lowering of the positive feedback current is observed. Thus, intuitively, the contrast is reduced as the object conductivity diminishes. Numerical simulations are necessary for more quantitative description of the dependence of the object conductivity on the SECM image contrast.

To correlate results of model experiments described above to the SECM imaging of iron inclusions in the $\mathrm{Nd}_{13.5} \mathrm{Fe}_{79.5} \mathrm{Si}_{1} \mathrm{~B}_{6}$ magnet, it should be emphasized that conducting objects (Fe inclusions) visualized by SECM (Fig. 6 and 8, the main text) are at least 1.5 times smaller in reality than they appear on SECM images. Moreover, the shape of real object might be quite different from that visible on SECM images. However, using SECM in feedback mode we were able to visualize objects with dimensions equal to dimensions of Fe-inclusions in $\mathrm{Nd}_{13.5} \mathrm{Fe}_{79.5} \mathrm{Si}_{1} \mathrm{~B}_{6}$. 


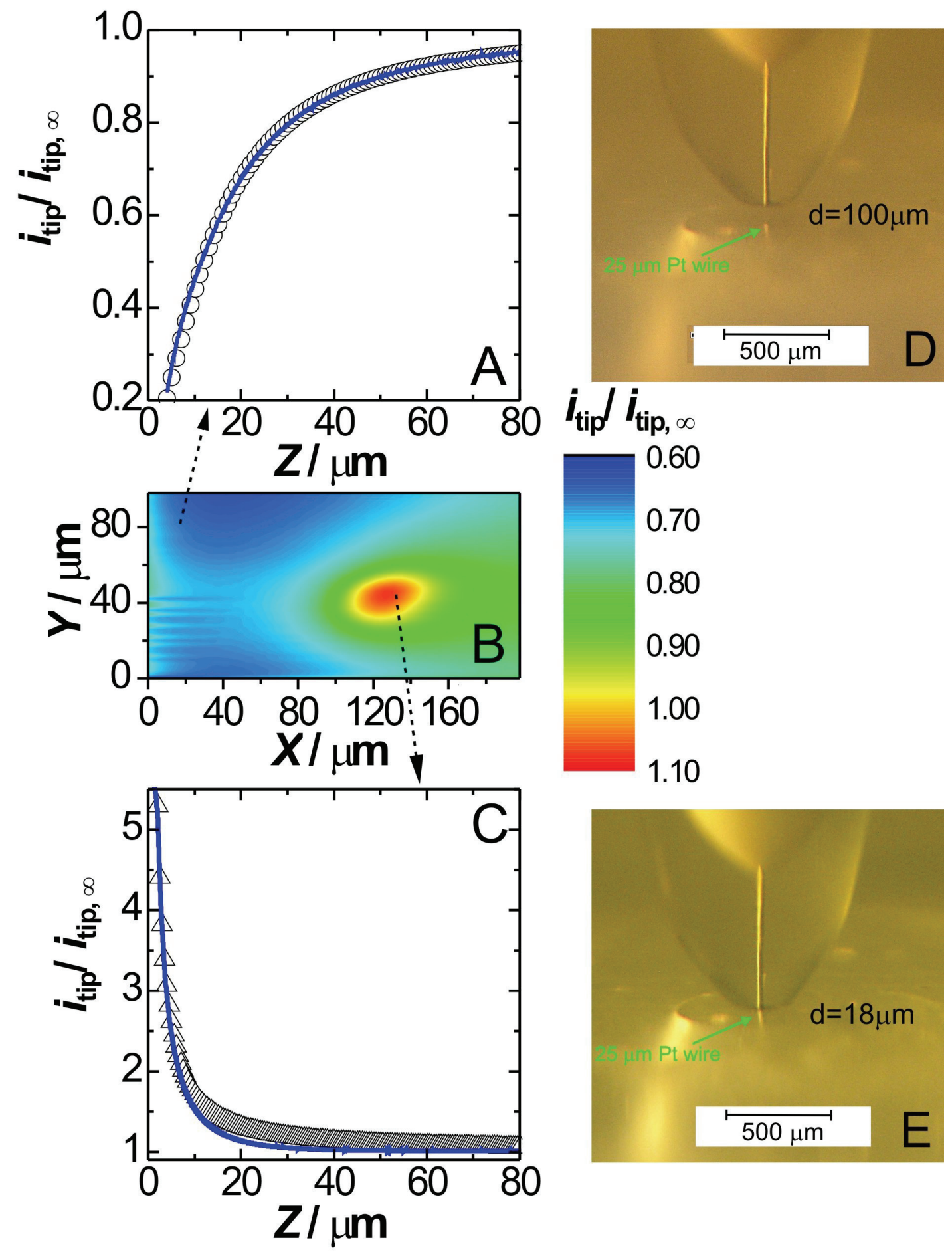

Fig. S-4. (A) An approach curve (blue line) recorded over an insulating glass sheath. (B) SECM image of the $25 \mu \mathrm{m}$ Pt wire embedded in glass recorded at $d=20 \mu \mathrm{m}$. (C) An approach curve recorded over the centre of Pt micro-wire. Tip movement rate during recording approach curves: $0.25 \mu \mathrm{m} \mathrm{s}^{-1}$. (D,E) optical micrographs confirming tip-substrate alignment during recording approach curves over $\mathrm{Pt}$ microvire. Electrolyte: $2 \mathrm{mmol} \mathrm{dm}{ }^{-3} \mathrm{FcCOOH}$ in $0.1 \mathrm{~mol} \mathrm{dm}^{-3}$ phosphate buffer at $\mathrm{pH}=7 . E_{t i p}=0.5 \mathrm{~V}$ vs SCE. $E_{\text {sub }}=-0.3 \mathrm{~V}$ vs SCE. Pt tip microelectrode diameter: $25 \mu \mathrm{m}(\mathrm{RG}=8)$. Theoretical approach curves for the negative feedback (A, circles) and the for positive feedback ( $\mathrm{C}$, triangles) were calculated on the basis of equations derived in Ref. [40]. Deviation of the approach curve recorded over Pt micro-wire from the theoretical curve at larger distances reflects the fact that the theoretical curve was calculated for the uniformly conducting substrate (not for a single conducting spot). At very short distances, tip- 
substrate system operates like thin-layer cell, and the experimental curve matches quite well the theoretical one.
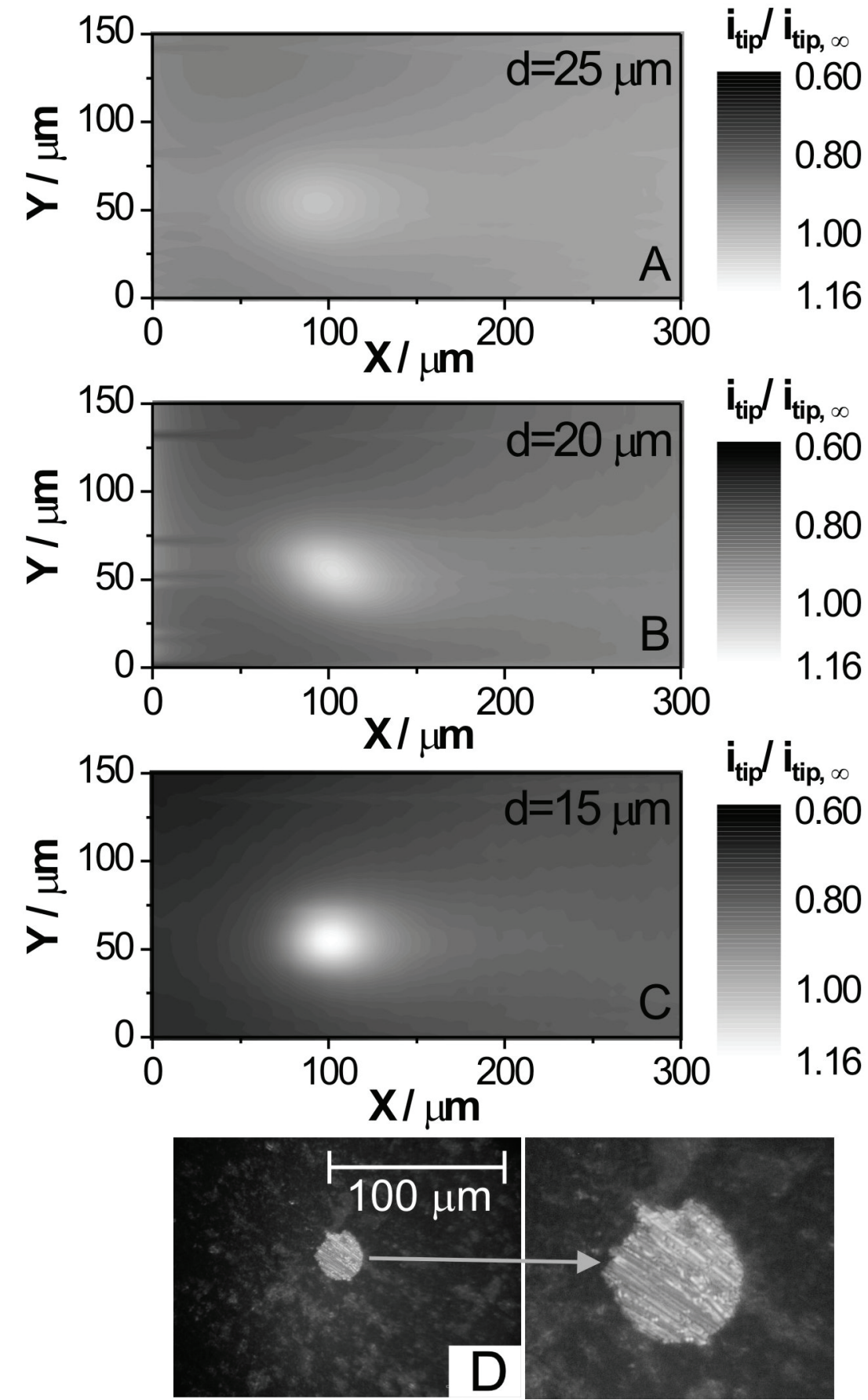

Fig. S-5. SECM images (A-C) as for Fig. S-3 but displayed in a gray scale. Scale color bars are identical in all three cases. DIC optical micrograph of the substrate (D) also in a gray scale. 


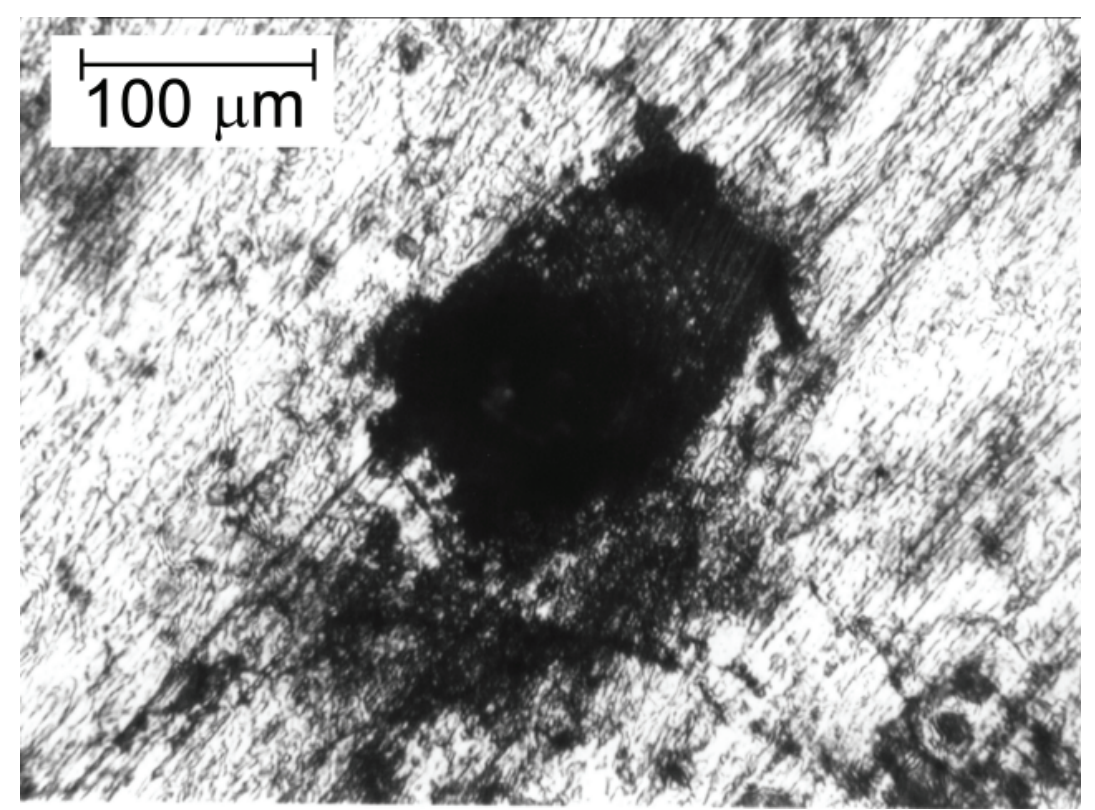

Fig. S-6. Optical microscopic image (in bright filed) of the pit developed at the position $\mathrm{X}=300 \mu \mathrm{m}$ (see Fig. 10, the main text). The image was obtained after removing corrosion products from the sample in an ultrasonic bath.

\section{Redox conductivity changes in the passive film formed on pure iron in phosphate buffer solutions}

Cyclic voltammograms recorded for iron electrode in $0.1 \mathrm{~mol} \mathrm{dm}^{-3}$ phosphate buffer solution (at $\mathrm{pH}=7$ ) and in $0.1 \mathrm{~mol} \mathrm{dm}^{-3}$ phosphate buffer solution (at $\mathrm{pH}=7+0.1 \mathrm{~mol} \mathrm{dm}^{-3} \mathrm{NaCl}$ ) are presented in Fig. S-7. It comes from Fig. S-7 that iron undergoes passivation in $0.1 \mathrm{~mol} \mathrm{dm}^{-3}$ phosphate buffer solution. The important feature of CVs for iron in phosphate buffer solution is the existence of a pair of redox peaks (indicated by blue arrows in Fig. S-7 A and B) in the passive region. The mid-peak potential of these redox peaks are $-0.302 \mathrm{~V}$ and $-0.284 \mathrm{~V}$ for the potential scan rates of $10 \mathrm{mV} \mathrm{s}^{-1}$ and 1 $\mathrm{mV} \mathrm{s}^{-1}$, respectively. The existence of oxidation-reduction peaks in the passive region implies that redox reaction occurs within a passive layer existing on iron in the phosphate buffer environment (because there are no any redox species in the solution). According to literature data [S3-S5], the most plausible redox processes, that may occur at the potentials of redox peaks labeled in Fig. S-7 A and B, are as follows:

$$
\begin{array}{ll}
\mathrm{Fe}(\mathrm{OH})_{2} \leftrightarrow \gamma-\mathrm{FeOOH}+2 \mathrm{H}_{2} \mathrm{O}+\mathrm{H}^{+}+\mathrm{e}^{-} & E=-0.41 \mathrm{~V} \text { vs SCE at } \mathrm{pH}=7 \\
\mathrm{Fe}_{3}\left(\mathrm{PO}_{4}\right)_{2}+6 \mathrm{H}_{2} \mathrm{O} \leftrightarrow 3 \gamma-\mathrm{FeOOH}+2 \mathrm{HPO}_{4}^{2-}+7 \mathrm{H}^{+}+3 \mathrm{e}^{-} & E=-0.10 \mathrm{~V} \text { vs SCE at } \mathrm{pH}=7
\end{array}
$$


Values of the redox potentials for reactions (S3) and (S4) have been taken from the Pourbaix diagram for iron in phosphate environment [S3].
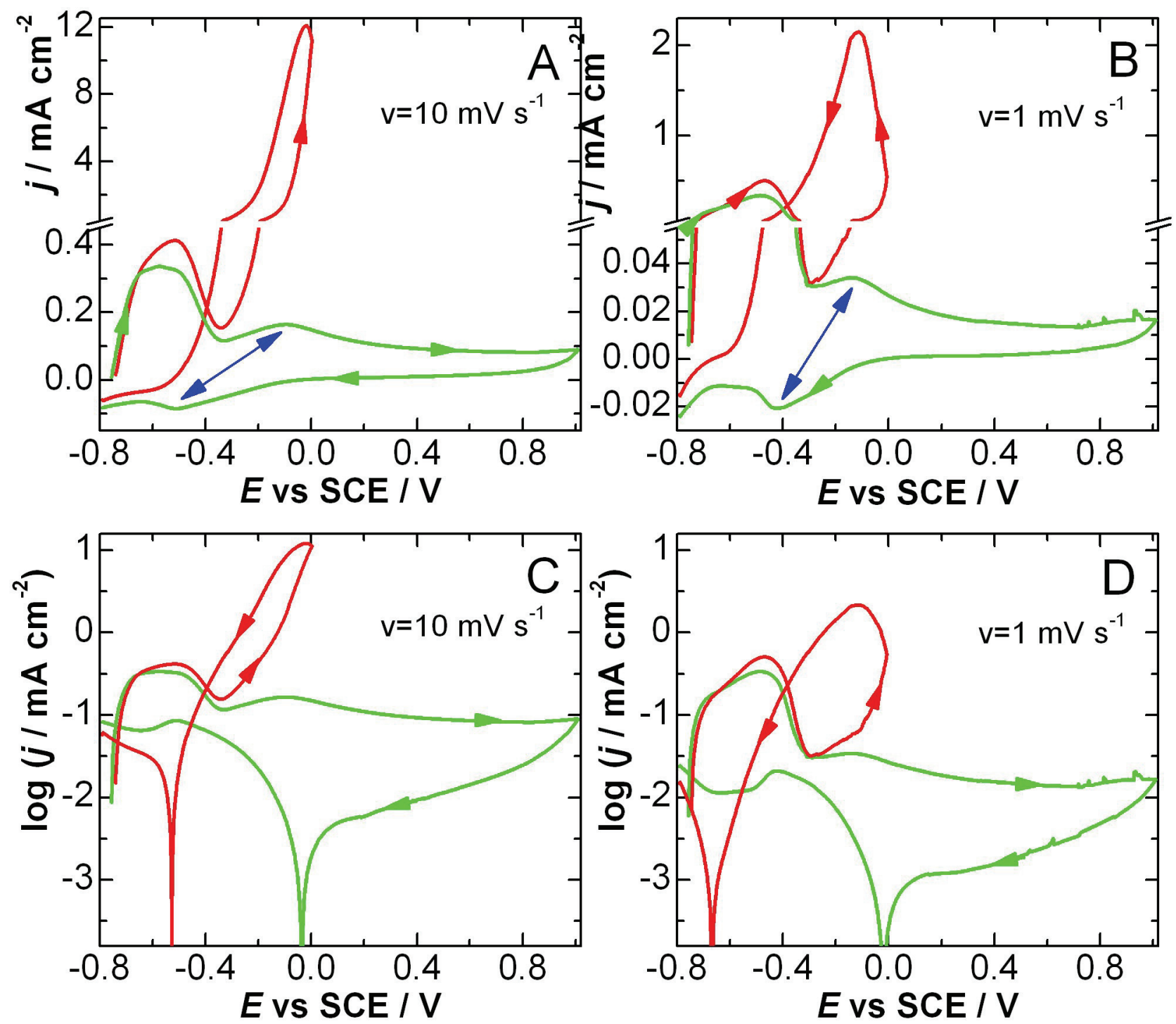

$-0.1 \mathrm{M}$ phosphate buffer $\mathrm{pH}=7+0.1 \mathrm{M} \mathrm{NaCl}$ $0.1 \mathrm{M}$ phosphate buffer $\mathrm{pH}=7$

Fig. S-7. Cyclic voltammograms and polarization curves recorded for iron electrode in $0.1 \mathrm{~mol} \mathrm{dm}^{-3}$ phosphate buffer solution at $\mathrm{pH}=7$ (green lines) and in $0.1 \mathrm{~mol} \mathrm{dm}^{-3}$ phosphate buffer solution at $\mathrm{pH}=7$ $+0.1 \mathrm{~mol} \mathrm{dm}^{-3} \mathrm{NaCl}$ (red lines). Scan rate: $10 \mathrm{mV} \mathrm{s}^{-1}(\mathrm{~A}, \mathrm{C})$ and $1 \mathrm{mV} \mathrm{s}^{-1}(\mathrm{~B}, \mathrm{D})$. Linear current density scale (A,B). Logarithmic current density scale (C,D). Cyclic voltammograms were recorded starting from open circuit potential that was reached after $5 \mathrm{~min}$ following the electrode immersion in appropriate solution.

Passive film on iron is a complex system consisting of many iron species (oxides, oxyhydroxides and phosphates of iron(II) and iron(III) [S5-S7]. In view of the present work, it should be emphasized that occurrence of any redox reaction in solid state would lead to changes in redox conductivity of the passive film on iron in the potential range where these redox reactions can take place. Redox 
conductivity becomes the highest when the ratio of concentrations of the reduced and oxidized forms is equal to unity, and this should happen at potentials close to the mid-peak potentials in cyclic voltammograms. When either the oxidized form or reduced form predominates, the redox conductivity lowers.

In order to compare changes in the conductivity of passive films on iron and the $\mathrm{Nd}_{13.5} \mathrm{Fe}_{79.5} \mathrm{Si}_{1} \mathrm{~B}_{6}$ magnet, we performed electrochemical impedance (EIS) measurements. Changes in the electrode admittance vs potential are presented in Fig. S-8. Assuming with large degree of approximation, that the system can be described by resistance and capacitance connected in parallel, the real part of admittance should reflect the passive film conductivity. Indeed, for the iron electrode, the curve a in Fig. S-8 reaches its maximum at the potential of $-0.24 \mathrm{~V}$ vs SCE which is close to the mid-peak potential (that could be obtained from cyclic voltammetry as discussed above). The redox conductivity of the passive film should be the highest at that potential. Moreover, the admittance curve obtained for the $\mathrm{Nd}_{13.5} \mathrm{Fe}_{79.5} \mathrm{Si}_{1} \mathrm{~B}_{6}$ electrode has monotonous course (no extreme is visible). These results clearly demonstrate existence of redox conductivity in the passive film on iron and, practically, lack of it in the passive film on the $\mathrm{Nd}_{13.5} \mathrm{Fe}_{79.5} \mathrm{Si}_{1} \mathrm{~B}_{6}$ magnet. It should be stressed that the passive film on the $\mathrm{Nd}_{13.5} \mathrm{Fe}_{79.5} \mathrm{Si}_{1} \mathrm{~B}_{6}$ magnet is composed of trivalent neodymium compounds (mostly $\mathrm{Nd}_{2} \mathrm{O}_{3}$ which is insulating and non-electroactive $[\mathrm{S} 8, \mathrm{~S} 9])$, and redox conductivity is not expected for such compounds (neodymium ions are only tri-valent).

The fact that redox conductivity of passive film on iron was potential-dependent might explain why we were able to observe iron inclusions in the $\mathrm{Nd}_{13.5} \mathrm{Fe}_{79.5} \mathrm{Si}_{1} \mathrm{~B}_{6}$ magnet by $\mathrm{SECM}$ in feedback mode after certain time following the sample immersion in the phosphate buffer solution containing chlorides. It should be rationalized that the potential of the $\mathrm{Nd}_{13.5} \mathrm{Fe}_{79.5} \mathrm{Si}_{1} \mathrm{~B}_{6}$ electrode increased ca. $0.1 \mathrm{~V}$ towards more positive values (i.e. from $-0.45 \mathrm{~V}$ to $-0.36 \mathrm{~V}$ vs SCE in Fig. 3 of the main text) after $56 \mathrm{~h}$ of immersion. This phenomenon most likely resulted in the increase of redox conductivity of the passive layer existing on iron inclusions. 


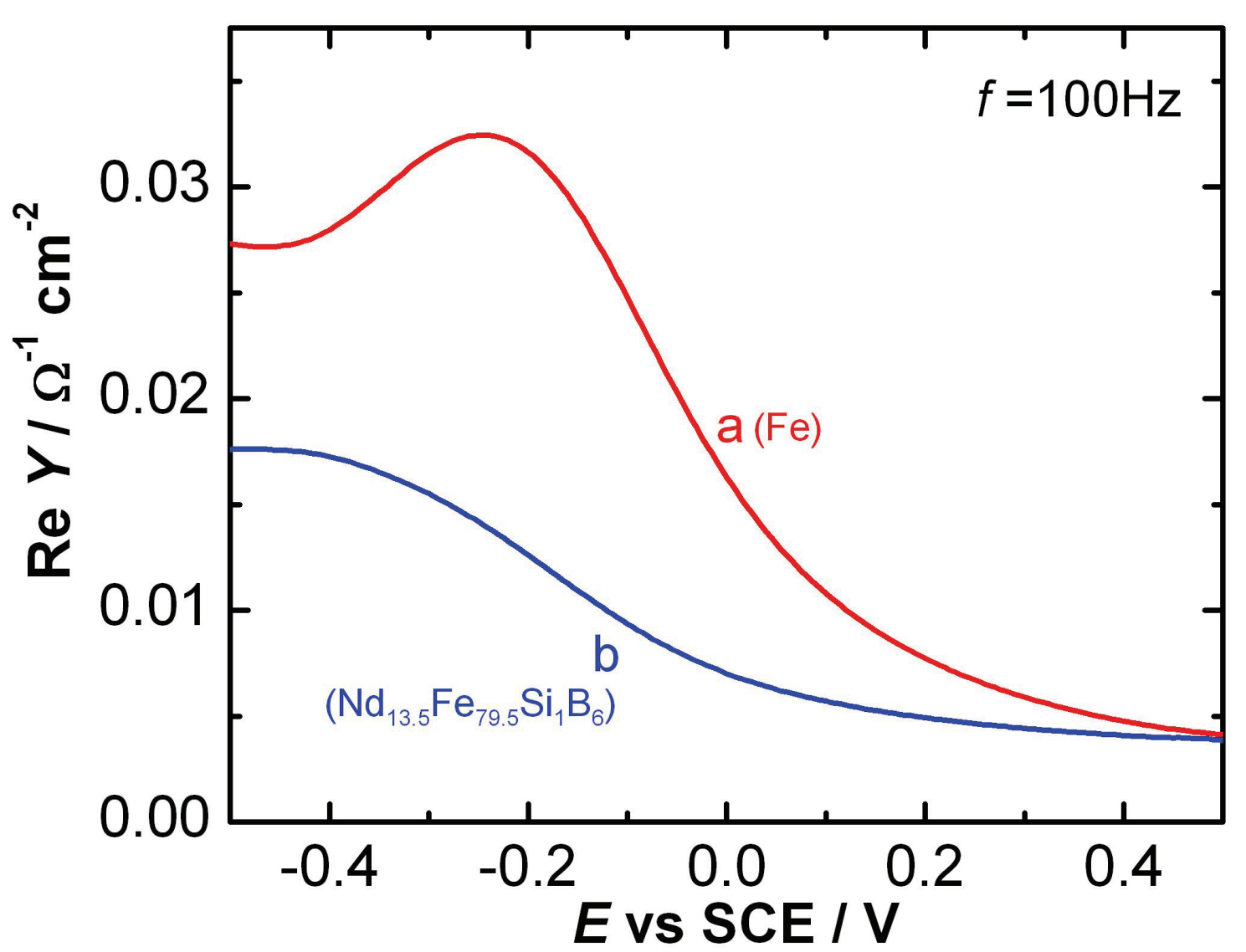

Fig. S-8. Plot of the real part of electrode admittance vs potential for iron electrode (a) and for $\mathrm{Nd}_{13.5} \mathrm{Fe}_{79.5} \mathrm{Si}_{1} \mathrm{~B}_{6}$ electrode (b) recorded at frequency, $f=100 \mathrm{~Hz}$ in $0.1 \mathrm{~mol} \mathrm{dm}^{-3}$ phosphate buffer solution. Before measurements, electrodes were kept at the potential $0.5 \mathrm{~V}$ vs SCE for 30 min in order to form the passive film rapidly. Potentials were decreased during measurements. EIS measurements were performed using CHI760B electrochemical workstation (CH Instruments, Austin, TX, U.S.A.). Platinum gauze was used as a counter electrode and $\mathrm{Ag} / \mathrm{AgCl}$ (in $1 \mathrm{~mol} \mathrm{dm}^{-3} \mathrm{NaCl}$ ) served as a reference electrode; but all presented potentials are recalculated to the SCE scale.

Potential dependent changes in the conductivity of the passive film formed on iron are also manifested in impedance spectra which were recorded during prolonged exposure of iron to the phosphate buffer solution (Fig. S-9A,B). The open circuit potential of iron (immersed in phosphate buffer) increases monotonically with time what is due to the passive layer growth (Fig. S-9C). It is noteworthy that, within the potential range between -0.76 and $-0.47 \mathrm{~V}$ vs SCE, the impedance modulus increases for the whole frequency range. Diminishing of the impedance modulus is observed for potentials larger than $-0.47 \mathrm{~V}$ up to the potential of $-0.29 \mathrm{~V}$ (the highest potential reached by iron electrode under open circuit conditions during our experiment). This fact suggests increase in the passive film conductivity. However, our EIS characterization of iron electrode should be considered only as the qualitative support of SECM results obtained for iron inclusions. The passive film on iron 

impedance spectra is beyond the scope of our work.
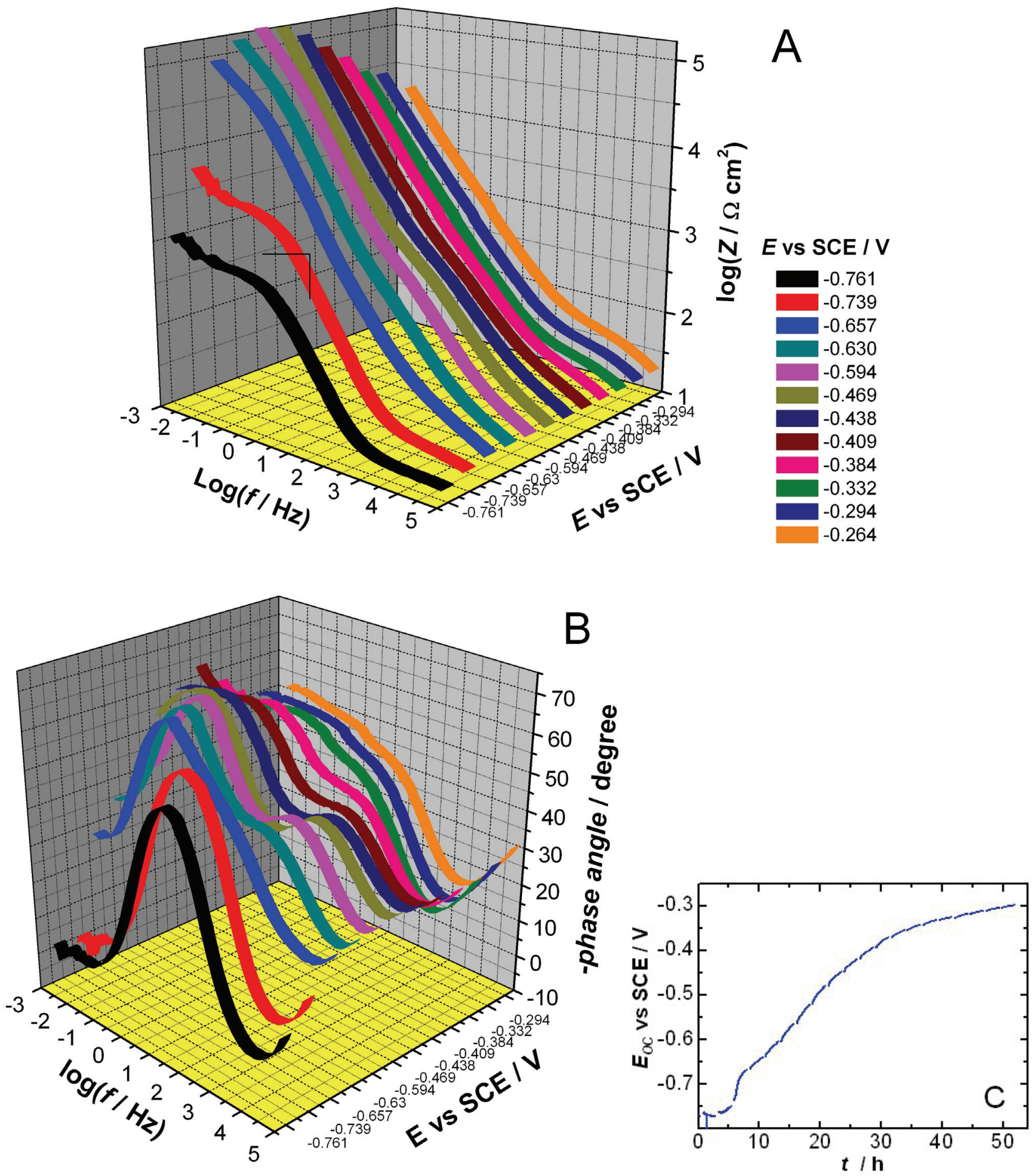

Fig. S-9. Electrochemical impedance spectra (Bode plots - A, B) recorded during prolonged immersions of iron electrode in $0.1 \mathrm{~mol} \mathrm{dm}^{-3}$ phosphate buffer solution at $\mathrm{pH}=7$. The iron sample was not biased for $2 \mathrm{~h}$, and during that period its open circuit potential $\left(E_{O C}\right)$ was continuously measured. The impedance spectrum was acquired at the potential equal to the $E_{O C}$ potential that was established after the $2 \mathrm{~h}$ period. This procedure was repeated 21 times. Changes of $E_{O C}$ occurring during this experiment are plotted in Fig. C. 


\section{REFERENCES}

(S1) Combellas, C.; Fermigier, M.; Fuchs, A.; Kanoufi, F. Anal. Chem. 2005, 77, 7966.

(S2) Pluta, M. Advanced Light Microscopy, vol. 1, Principles and basic concepts; PWN-Elsevier: Warsaw-Amsterdam; 1988; (a) pp 339-344; (b) pp 133-134, 345-346.

(S3) Giacomelli, C.; Giacomelli, F. C.; Bortolluzzi, R. L.; Spinelli, A. Anti-Corros. Methods Mater. 2006, 53, 232.

(S4) Giacomelli, C.; Spinelli, A. Anti-Corros. Methods Mater. 2004, 51, 189.

(S5) Martini, E. M. A.; Amaral, S. T.; Muller, I. L. Corros Sci. 2004, 46, 2097.

(S6) Benzakour. J.; Derja, A. J. Electroanal. Chem. 1997, 437, 119.

(S7) Sieber, I. V.; Hildebrand, H.; Virtanen, S.; Schmuki, P. Corros. Sci. 2006, 48, 3472.

(S8) Dakhel, A. A. J. Alloy. Compd. 2004, 376, 38.

(S9) Song, M.-K.; Rhee, S.-W. Thin Solid Films, 2005, 492, 19. 